\title{
Milan à Albenga. La coupole perdue du baptistère milanais San Giovanni alle Fonti : un essai de restitution
}

\section{Alžběta Filipová}

\section{(2) OpenEdition}

\section{Journals}

Édition électronique

URL : http://journals.openedition.org/edl/1140

DOI : $10.4000 /$ edl. 1140

ISSN : 2296-5084

Éditeur

Université de Lausanne

\section{Édition imprimée}

Date de publication : 15 mai 2018

Pagination : 85-104

ISBN : 978-2-940331-68-0

ISSN : 0014-2026

\section{Référence électronique}

Alžběta Filipová, « Milan à Albenga. La coupole perdue du baptistère milanais San Giovanni alle Fonti un essai de restitution », Études de lettres [En ligne], 2 | 2018, mis en ligne le 15 mai 2020, consulté le

11 décembre 2020. URL : http://journals.openedition.org/edl/1140 ; DOI : https://doi.org/10.4000/edl. 1140 


\section{MILAN À ALBENGA \\ LA COUPOLE PERDUE DU BAPTISTÈRE MILANAIS SAN GIOVANNI ALLE FONTI: UN ESSAI DE RESTITUTION}

Cet article propose une hypothèse de restitution de la composition de la coupole du baptistère épiscopal milanais de San Giovanni alle Fonti, disparu depuis presque six siècles. Cette dernière se base sur une analyse des liens qui unissent Milan et ce qui nous est parvenu de l'édifice baptismal d'origine (fragments des murs périphériques, éléments de la décoration) avec le baptistère d'Albenga, encore conservé, qui peut être considéré comme une véritable copie du modèle milanais. Une attention particulière est portée à l'interprétation de la mosaïque qui orne la niche orientale à Albenga, dont l'élément central pouvait, à notre avis, couronner la décoration de la coupole à Milan.

Peu d'éléments subsistent aujourd'hui du baptistère majeur de la Milan tardo-antique, San Giovanni alle Fonti, qui faisait jadis partie du complexe épiscopal milanais, près de l'abside de la basilica maior ${ }^{1}$. Le reste des murs de l'édifice octogonal, ainsi que de rares éléments de la décoration suggèrent néanmoins que ce baptistère était non seulement couronné d'une coupole, mais que celle-ci était ornée de précieuses mosaïques en verre ${ }^{2}$. Tandis que la forme architecturale originelle du baptistère peut être restituée grâce à ses antécédents locaux et grâce à d'autres édifices baptismaux qui s'en sont sans doute inspirés, aucune restitution de sa décoration primitive en mosaïque n’a été tentée jusqu’à

I. Cf. L. Fieni, «Il battistero di San Giovanni alle Fonti di Milano»; S. Lusuardi Siena, «II complesso episcopale di Milano».

2. S. Lusuardi Siena, E. Dellù, M. L. Delpiano, E. Monti, «Lettura archeologica e prassi liturgica nei battisteri ambrosiani tra IV e VI secolo»; E. Neri, M. Verità, A. Conventi, "Glass mosaic tesserae from the 5th to 6th century baptistery of San Giovanni alle Fonti, Milan, Italy». 
présent. Cet article propose donc de combler cette lacune en exposant les liens du baptistère milanais avec l'un des rares édifices baptismaux paléochrétiens ayant préservé sa décoration originelle: le baptistère d'Albenga, en Ligurie. Il s'agit du monument clé pour la compréhension de la relation complexe entre Milan, métropole ecclésiastique de l'Italia Annonaria dès l'époque de l'évêque Ambroise (374-397) ${ }^{3}$, et les évêchés suffragants, où les idées venues de la métropole trouvent souvent d'intéressantes reprises ${ }^{4}$. Les «idées» en question concernent tant la politique ecclésiastique, le culte des saints et de leurs reliques, que la construction des lieux de culte et leur décoration. À travers la diffusion du culte des saints locaux, miraculeusement "inventés" par Ambroise de Milan en $386^{5}$, l'évêque milanais orchestre un véritable transfert culturel vers les évêchés dans la "sphère d'influence" milanaise et crée ainsi un territoire ecclésiastique unifié. Dans cette perspective, il est à notre avis possible d'appréhender la mosaïque qui orne la niche orientale du baptistère d'Albenga - représentant une image trinitaire hautement symbolique et mentionnant la présence des reliques des saints ambrosiens - en tant que remploi conceptuel de la mosaïque milanaise. Pour soutenir cette hypothèse, nous examinerons plusieurs liens qui unissent la ville d'Albenga avec Milan et chercherons à mettre en évidence la parenté culturelle qu'entretient le baptistère monumental ligure avec le baptistère épiscopal milanais.

\section{San Giovanni alle Fonti, données archéologiques}

Avant d'aborder la question de la mosaïque qui ornait jadis sa coupole, il est nécessaire tout d'abord de considérer les restes archéologiques du baptistère milanais et, sur la base de l'analyse de ces derniers, de rappeler

3. Cf. la formation de la métropole ecclésiastique milanaise à l'époque d'Ambroise, E. Cattaneo, Terra di Sant'Ambrogio; R. Lizzi, "Ambrose's contemporaries and the christianization of northern Italy».

4. Cf. ma thèse de doctorat soutenue en avril 2017 à l'Université de Lausanne, qui est en cours de publication par la maison d'édition romaine Viella. A. Filipová, Milan sans frontières. Le culte et la circulation des reliques milanaises et leur impact sur la diffusion de l'art et l'architecture de Milan pendant l'Antiquité tardive.

5. Ambroise de Milan, Épître 77, p. 154 sq. Une description et une analyse des évènements sont faites par J. San Bernardino, "Sub imperio discordiae». 


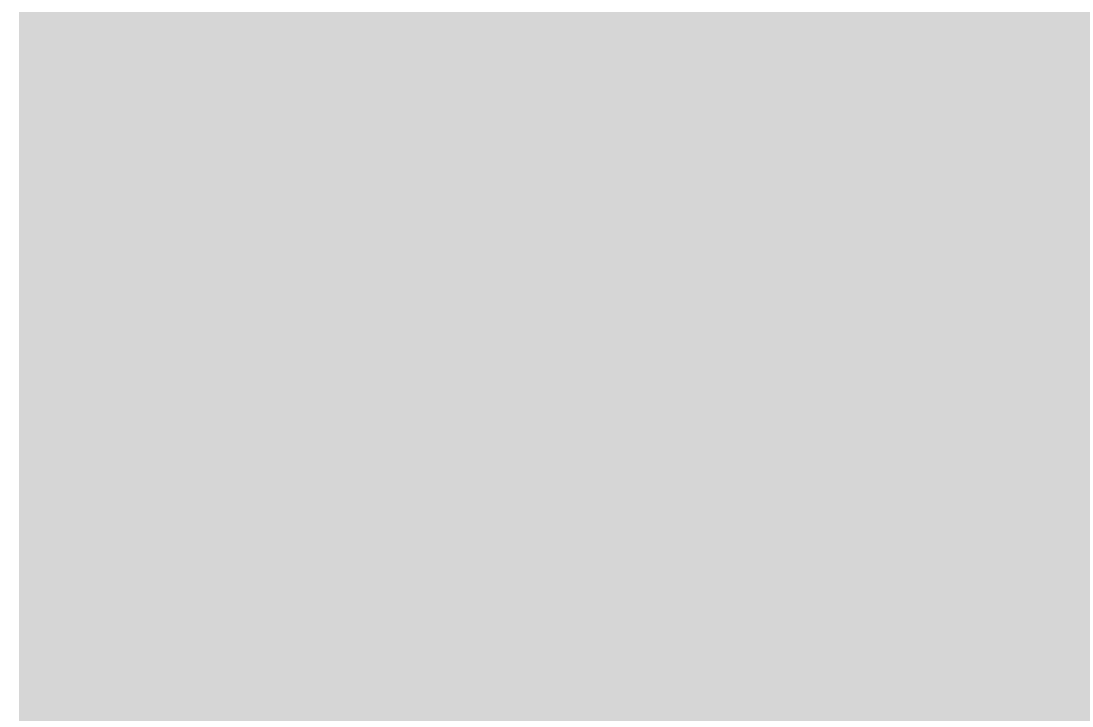

Fig. 1 - Milan, San Giovanni alle Fonti, restitution du plan de l'édifice.

l'hypothèse de restitution de la forme architecturale de l'édifice. Ce dernier a été démoli sans doute avant 1455 pour faire place à un espace ouvert devant le Dôme, dont la construction a commencé en $1386^{6}$. La campagne de fouilles archéologiques, conduite entre 1961 et 1962, a toutefois mené à la découverte des restes du baptistère paléochrétien.

La structure découverte présente un plan octogonal à l'extérieur (les côtés font 7,4 m, le diamètre est de 19,3 m) avec des niches creusées dans l'épaisseur du mur, semi-circulaires et quadrangulaires en alternance (fig. 1). En correspondance avec les quatre niches rectangulaires s'ouvraient quatre portes ${ }^{7}$. Les murs de l'édifice sont conservés plus ou moins jusqu'à 2,4 m de hauteur. Les bases des colonnes intérieures sont posées aux angles d'un octogone: soit elles étaient en relation avec le système d'articulation en élévation (ou directement de couverture), soit elles ne remplissaient qu'une fonction décorative. La seconde solution est connue du mausolée de Dioclétien à Split, ou, plus tard, est employée

6. S. Lusuardi Siena, «Il complesso episcopale», p. 106.

7. Ibid., p. 109 sq. 
Fig. 2 - Milan, San Giovanni alle Fonti, restitution de l'édifice en élévation. 
dans le baptistère des Orthodoxes à Ravenne ${ }^{8}$ (fig. 2). Il s'agit cependant d'une solution architecturale qui entre parfaitement dans la lignée de la tradition des édifices octogonaux milanais, avec les mausolées impériaux de Saint-Victor-au-corps d'un côté et de Saint-Aquilin de l'autre? Des éléments de l'aménagement intérieur du baptistère se sont également conservés: notons les fonts baptismaux octogonaux, de 5,5 $\mathrm{m}$ de diamètre, qui se trouvent au milieu de la structure, et les restes d'un pavement en opus sectile. De plus, de nombreuses tesselles et fragments de mosaïques murales ont été retrouvés pendant les fouilles et ont récemment fait objet d'analyses chimiques et techniques ${ }^{10}$. Les 271 fragments de mosaïque retrouvés ne représentent cependant qu'un pourcentage de la quantité des tesselles qui ornaient jadis la surface de la coupole, et ne permettent donc pas de décrire les décorations de cette dernière.

En ce qui concerne la datation du monument, plusieurs hypothèses ont été formulées: en proposant un moment soit précédant l'épiscopat d'Ambroise (autour de 350) ${ }^{11}$, soit des années de son épiscopat. Cette hypothèse est traditionnellement démontrée sur la base des vers de dédicace attribués à Ambroise et transcrits dans le Sylloge de Lorsch ${ }^{12}$. Les données archéologiques laissent d'ailleurs supposer une datation de la première structure à la seconde moitié $\mathrm{du}_{\mathrm{IV}}^{\mathrm{e}}$ siècle $^{13}$. Il faut néanmoins discerner au moins deux étapes dans la construction du baptistère milanais. Selon l'état actuel des connaissances, l'édifice conserve, dans la planimétrie et dans les restes des murs périphériques, les structures de l'époque ambrosienne ou pré-ambrosienne, tandis que les fonts baptismaux centraux, le pavement et les revêtements des parois en opus sectile, ainsi que probablement la décoration en mosaïque et une nouvelle coupole, sont à assigner aux interventions de l'évêque Laurent I $^{\text {er }}$

8. S. Lusuardi Siena, F. Sacchi, "Gli edifici battesimali di Milano e di Albenga», p. 681 et 690 .

9. Cf. M. J. Johnson, The Roman imperial mausoleum in Late Antiquity, p. 156-167.

Io. Cf. E. Neri, M. Verità, A. Conventi, «Glass mosaic tesserae from the 5 th to 6 th century baptistery of San Giovanni alle Fonti, Milan, Italy».

II. D. Kinney, «Le chiese paleocristiane di Mediolanum», p. 54-57.

I2. Vatican, Biblioteca Apostolica, Vat. MS Pal. Lat. 833. Les distiques se trouvent transcrits dans $C I L$ V, 2, 2, p. 617. L'attribution ambrosienne, généralement acceptée, a été proposée pour des motifs philologiques par G. B. Pighi, "Commentariolus electorum».

I3. S. Lusuardi Siena, M. Sannazaro, «I battisteri del complesso episcopale milanese alla luce delle recenti indagini archeologiche». 
(489-511). Cette hypothèse se base sur les vers de l'hymne In Baptisterio mediolanensi d'Ennode de Pavie ${ }^{14}$. La datation des tesselles de verre produites approximativement au tournant $\mathrm{du} \mathrm{VI} \mathrm{V}^{\mathrm{e}}$ siècle peut également être interprétée en ce sens ${ }^{15}$. Il est ainsi probable que la structure monumentale de la fin du IV ${ }^{\mathrm{e}}$ siècle soit restée pratiquement inchangée, au moins au niveau du rez-de-chaussée, mais il est plausible que le niveau supérieur ait subi des interventions, avec notamment le rétrécissement de la couverture centrale. Selon Silvia Lusuardi Siena et Furio Sacchi, cette nouvelle solution architecturale aurait été reprise également à Albenga ${ }^{16}$.

\section{Le baptistère d'Albenga et sa relation à Milan}

Le baptistère d'Albenga est l'unique édifice baptismal en Ligurie encore conservé dans son élévation et, comme mentionné, l'un des rares qui porte, au moins en partie, sa décoration mussive d'origine ${ }^{17}$. Au niveau architectural, le baptistère a un plan octogonal, avec les côtés partiellement désaxés, une symétrie octogonale est toutefois soulignée par l'alternance de huit niches semi-circulaires ou rectangulaires, insérées dans l'épaisseur des murs et présentant chacune une fenêtre (fig. 3). Les arcs, au-dessus des ouvertures des niches, sont soutenus par huit colonnes. Au centre de l'édifice se trouvent des fonts baptismaux octogonaux. Au-dessus de la partie centrale de l'édifice s'élève, plus ou moins à partir du milieu de l'élévation, le tambour avec une arcature maçonnée. Le tambour est aujourd'hui couvert d'un plafond en charpente. Toutefois, jusqu'au début du $\mathrm{XX}^{\mathrm{e}}$ siècle, moment de sa démolition, une coupole couronnait le sommet du baptistère ${ }^{18}$.

Sans entrer dans les détails, même cette brève description suggère une étroite relation entre ce baptistère et celui de Milan: il s'agit surtout de l'emploi d'une articulation architecturale semblable - malgré les

I4. Ennode de Pavie, Carmina, II, 56.

15. E. Neri, M. Verità, A. Conventi, "Glass mosaic tesserae from the 5th to 6th century baptistery of San Giovanni alle Fonti, Milan, Italy».

16. S. Lusuardi Siena, F. Sacchi, "Gli edifici battesimali di Milano e di Albenga", p. 698.

17. Pour la bibliographie, voir la monographie de M. Marcenaro, Il battistero «monumentale» di Albenga.

I8. Cf. M. Marcenaro, «La cupola del Battistero di Albenga». 
Fig. 3 - Albenga, baptistère, plan de l'édifice.

dimensions réduites et l'exécution quelque peu irrégulière à Albenga. Quel est cependant l'arrière-plan de cette reprise? Au niveau ecclésiastique, dès l'instauration de son évêché en 451, la cité d'Albenga a été sous la juridiction de la métropole milanaise. Du milieu du $\mathrm{V}^{\mathrm{e}}$ siècle est également datée la première phase de construction du baptistère épiscopal, qui comprend surtout la structure murale ${ }^{19}$. Toutefois, comme à Milan, les archéologues ont discerné une seconde phase de construction, de la fin $\mathrm{du} \mathrm{V}^{\mathrm{e}}$ siècle, qui comprend un nouveau pavement en opus sectile et,

19. Cf. dernièrement O. Brandt et al., «Photo modelling as an instrument for stratigraphic analysis of standing buildings ». 
Fig. 4 - Albenga, baptistère, détail de l'inscription en mosaïque, fin $V^{\mathrm{e}}$-début $\mathrm{VI}^{\mathrm{e}}$ siècle.

selon toute vraisemblance, l'intégralité de la décoration en mosaïque ${ }^{20}$. La restauration et la nouvelle décoration auraient donc été parallèles aux travaux menés à Milan à la même période. Mais c'est également la mosaïque se trouvant dans et autour de la niche principale, en face de l'entrée, qui nous donne une clé pour comprendre la relation avec Milan.

Cette mosaïque présente une frise décorative qui inclut une inscription (fig. 4). Celle-ci peut être traduite de la manière suivante: "Ici sont les reliques d'Étienne, Jean Évangéliste, Laurent, Nabor, Felix, Gervais et Protais » ${ }^{21}$. Comme postulé dans ma thèse de doctorat, Milan était à la fois le lieu clé dans la diffusion de la pratique d'insertion des reliques dans les autels et le "producteur" le plus important de tels «objets sacrés» dès la fin du IV $\mathrm{IV}^{\mathrm{e}}$ siècle ${ }^{22}$. De fait, le plus célèbre évêque

20. D. Gandolfi, A. Frondoni, "Recenti indagini archeologiche nel battistero “monumentale" di Albenga.", p. 588 sq.

2I. NAMUS QUORUM HIC RELIQUIAE SUNT / STEFANI S.IOHANNIS LAURENTI NAVORIS PROTASI / EVANGEL- FELICIS GERVASI. Cette transcription, ainsi que la lecture des abréviations difficiles à lire, ont été faites en premier par P. Toesca, La pittura e la miniatura nella Lombardia, p. 22.

22. Cf. A. Filipová, Milan sans frontières. 
de Milan, Ambroise, et certains de ses successeurs, ont distribué les reliques secondaires des saints milanais Gervais et Protais (et dans une moindre mesure de Nabor et Felix, mais probablement aussi les reliques de contact d'autres saints encore) dans une quantité sans précédent et sur une aire de répartition géographique très étendue ${ }^{23}$. La présence des reliques des martyrs milanais a été documentée à travers tout le territoire de l'ancienne Gaule et du nord de l'Italie, mais aussi au sud de l'Italie et au nord de l'Afrique. Déjà Neil B. McLynn parlait de «stratégie de marketing" employée par Ambroise en vue de rehausser le prestige du siège épiscopal milanais, en rivalité avec Rome à la fin du IVe siècle ${ }^{24}$. À cette période, Milan servait de siège impérial, ce qui a contribué, avec le charisme d'Ambroise, à faire de la ville l'un des évêchés majeurs de l'Église universelle.

Dans la pensée théologique tardo-antique et médiévale, les reliques d'un même saint établissaient un lien privilégié entre les communautés qui les possédaient ${ }^{25}$. Comme nous pouvons le voir sur cet exemple concret évoquant la présence des reliques ambrosiennes sur la mosaïque, Albenga déclare son lien privilégié avec Milan. De surcroît, d'un point de vue archéologique, en se basant sur les trouvailles faites lors des fouilles, l'opinion admise par la plupart des chercheurs est que les reliques annoncées dans l'inscription ont été originellement placées dans le bassin rectangulaire creusé dans le sol de la niche mosaïquée ${ }^{26}$. Aucune trace de reliques ou de leur conteneur n'y a malheureusement été retrouvée, mais il est à notre avis signifiant qu'à l'époque où la mosaïque a été conçue, l'inscription susmentionnée confirme la présence de quatre saints milanais.

\section{La mosaïque à Albenga}

Mais qu'en est-il du reste de la décoration? Peut-on trouver également des parallèles avec Milan? Dans la lunette au-dessus de la fenêtre, une croix gemmée est représentée au centre, juchée des deux côtés par un

\footnotetext{
23. Cf. la synthèse de A. Filipová, "The circulation of blood, clay and ideas».

24. Cf. N. B. McLynn, Ambrose of Milan, p. 284.

25. Cf. G. Clark, "Victricius of Rouen".

26. M. Marcenaro, Il battistero "monumentale» di Albenga, p. 165.
} 
Fig. 5 - Albenga, baptistère, voûte mosaïquée de la niche orientale, fin $\mathrm{V}^{\mathrm{e}}$-début $\mathrm{VI}^{\mathrm{e}}$ siècle. 
agneau dans un pré fleuri. À l'intérieur de la voûte, la mosaïque présente un triple chrisme qui apparaît dans un ciel étoilé (fig. 5). Le chrisme est formé par des tesselles jaunes et blanches, et il est inscrit dans un champ circulaire composé de trois bandes d'un bleu graduellement plus foncé, avec trois fois les lettres Alpha et Oméga. Par un jeu subtil de couleurs, les trois bandes de cette composition sont unies en une seule image aux frontières perméables. Cette composition est entourée de douze colombes et couronnée par une petite croix. Contrairement à la scène bucolique qui représente un type de décoration commun dans le contexte baptismal ${ }^{27}$, le triple chrisme est un unicum. Il s'agit là d'une image symbolique de la Trinité, dont la présence, de prime abord, n'est pas surprenante dans un espace baptismal: le baptême du Christ est lui-même présenté dans les évangiles comme la première apparition de la Trinité.

Néanmoins, la clé pour comprendre cette composition, répétant trois fois le nom du Christ, est à chercher dans le rite baptismal et sa signification, tel que décrit notamment par Ambroise de Milan. Nathan Dennis a récemment développé la question en détail en proposant que l'iconographie de la mosaïque d'Albenga fonctionne comme paradigme pour comprendre la relation entre l'architecture et le mouvement du corps dans l'espace baptismal ${ }^{28}$. En effet, dans le christianisme ancien, l'union mystique entre le monde tangible et le surnaturel était particulièrement évidente lors du rite baptismal: le catéchumène était censé, grâce à la transformation de ses sens corporels en perception spirituelle, pouvoir accéder à la vision de Dieu ${ }^{29}$. Le corpus des catéchèses mystagogiques de la fin du $\mathrm{IV}^{\mathrm{e}}$ et du début du $\mathrm{V}^{\mathrm{e}}$ siècle indique, selon Vladimir Ivanovici, que le baptême a été conçu et orchestré comme une théophanie ${ }^{30}$. C'est pourquoi l'espace intérieur du baptistère était transformé au moment du rite en limen, le seuil entre la réalité physique et métaphysique, où le catéchumène entrevoit l'immatériel tout en restant enraciné dans le contexte matériel. Selon N. Dennis, le Chi-Rho à la fois triple et unique d'Albenga constitue un pendant visuel à l'interpénétration des réalités terrestre et céleste qui se produit pendant le rite baptismal ${ }^{31}$. Cette

\footnotetext{
27. Cf. N. S. Dennis, Performing paradise in the early Christian baptistery.

28. N. S. Dennis, "Bodies in motion».

29. Ambroise de Milan, De mysteriis, 3, 15, p. 162 sq. Cf. P. L. Gavrilyuk, S. Coakley (eds), The spiritual senses, p. 20-120.

30. Cf. V. Ivanovici, Manipulating theophany, p. 19-56.

3I. N. S. Dennis, «Bodies in motion».
} 
interaction avec le divin - à la fois à travers un mouvement imaginé et la procession réelle - est mise en relation avec la confession trinitaire du catéchumène, acte central de sa triple immersion dans les fonts ${ }^{32}$ et sa transformation en une véritable imago Trinitatis $^{33}$.

Ce qui demeure toutefois étonnant est le fait que la Trinité soit exprimée à Albenga à travers la triple répétition du nom du Christ. Pour résoudre ce problème, Mario Marcenaro a suggéré que la mosaïque a été conçue en tant que déclaration antiarienne au moment où les Ostrogoths ariens dominaient la majeure partie du nord de l'Italie après $492^{34}$. Une décoration mettant une telle emphase sur la divinité du Christ pourrait en effet être comprise comme une claire opposition à la pensée arienne, selon laquelle, en extrême synthèse, le Christ n'atteint pas la plénitude du Père, car il est engendré par celui-ci et, par conséquent, lui est postérieur ${ }^{35}$.

Néanmoins, à notre avis, la décoration représente plus qu'une prise de position théologique contre l'arianisme. Selon la description du rite baptismal donnée par Ambroise de Milan, avant de descendre dans les fonts baptismaux et avant de réciter la formule trinitaire, tout le corps du catéchumène a été oint par l'huile sainte pour qu'il devienne «l'athlète

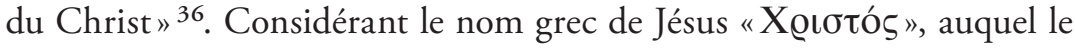
Chi-Rho fait référence et qui signifie "oint", l'onction du catéchumène renforce encore davantage son union mystique avec le Christ pendant le baptême. Déjà dans l'épître de saint Paul aux Galates, il est écrit: "Car vous êtes tous fils de Dieu par la foi en Jésus-Christ; vous tous, qui avez été baptisés en Christ, vous avez revêtu Christ " ${ }^{37}$. Enfin, dans une des catéchèses mystagogiques, saint Ambroise explique:

Dieu t’a donc oint, le Christ t’a marqué d'un signe. Comment? Tu as été marqué du signe de sa croix, du signe de sa passion. Tu as reçu ce signe pour lui ressembler, afin que tu ressuscites à son image. [...] Tu trouves ensuite ailleurs ceci de particulier: c'est Dieu qui t’a appelé,

32. Ambroise de Milan, De sacramentis, II, 20, p. 84-87; De mysteriis, V, 28, p. 170 sq.

33. N. S. Dennis, "Bodies in motion».

34. M. Marcenaro, Il battistero "monumentale» di Albenga, p. 129-191.

35. Pour la théologie arienne de la Trinité, voir par exemple L. Ayres, Nicaea and its legacy.

36. Ambroise de Milan, De sacramentis, I, 2, 4, p. 60-63.

37. Gal. 3, 26-27. 
tandis qu'au baptême c'est avec le Christ que tu as été crucifié d'une manière spéciale, et ensuite, quand tu reçois d'une manière spéciale le signe spirituel, tu vois qu'il y a distinction de personnes, mais que tout le mystère de la Trinité s'y enchaîne ${ }^{38}$.

Nous croyons ainsi que le triple chrisme correspond particulièrement bien au signe spirituel décrit par Ambroise, dont le néophyte est à jamais marqué et qu'il voit clairement déployé dans la niche orientale en sortant des fonts baptismaux au baptistère d'Albenga.

Il est évident qu'à Albenga, nous sommes en face d'une décoration très subtile au niveau théologique. Il y a quelques décennies, Carlo Bertelli avait suggéré que cette composition unique et très significative ne pouvait pas avoir été inventée pour le lieu où elle se trouve à présent. Sans qu'il n'ait jamais exposé des arguments qui l'auraient poussé à une telle suggestion, C. Bertelli avait imaginé que l'impulsion derrière l'élément central de la décoration devait être la réception d'une décoration monumentale bien plus importante et avait proposé comme modèle hypothétique le baptistère du Dôme de Milan ${ }^{39}$.

\section{La coupole de San Giovanni alle Fonti}

À la lumière de ce qui précède, nous voudrions donc exposer des arguments en faveur de cette hypothèse et proposer, de plus, une restitution hypothétique de l'élément central de la coupole du baptistère milanais.

Le premier argument concerne l'exécution et les matériaux utilisés pour la mosaïque à Albenga. Les analyses confirment une absence quasi totale de tesselles en or (le chrisme et la croix gemmée étant réalisés en tesselles jaunes bien moins coûteuses) et l'usage exhaustif de tesselles faites de verre refondu et recyclé ${ }^{40}$. Ceci témoigne sans doute du budget limité dont devait disposer un petit évêché pour la construction et la décoration de son baptistère monumental. Ceci est confirmé également par le fait qu'à l'intérieur du baptistère, seule la niche orientale devait être ornée de mosaïques, malgré le fait que la structure était couronnée d'une

38. Ambroise de Milan, De sacramentis, VI, 2, 7-8, p. 138-141.

39. C. Bertelli, «Mosaici a Milano», p. 334.

40. E. Franceschi, D. Nole, S. Vassallo, «Il mosaico del battistero di Albenga». 
coupole $^{41}$. La splendeur de l'édifice est maintenue par sa forme octogonale (quoique quelque peu irrégulière), sa coupole et ses mosaïques, mais avec des limites économiques évidentes. C'est pourquoi, en parlant d'une reprise "économique" d'un point de vue technique et matériel, il nous semble logique de penser qu'une composition si sophistiquée et élaborée, combinant des aspects théologiques, rituels et peut-être également politiques, soit le fruit d'importation d'un centre intellectuel et ecclésiastique de première importance.

Ne voulant pas nourrir davantage le paradigme historiographique "centre-périphérie", parfois trop simpliste, nous tenons à présenter un second argument qui concerne la composition même de la mosaïque. En regardant le motif central circulaire entouré d'étoiles, le tout disposé sur une surface courbe quadrangulaire, nous ne pouvons pas nous empêcher de constater une certaine dissonance visuelle. Le disque avec le chrisme, entouré de colombes, tout comme les étoiles alignées, semble être écrasé par la frise décorative qui encadre la voûte (fig. 5). Cette dissonance nous pousse à croire qu'à Albenga, nous sommes en face de l'adaptation à un petit espace, pour des raisons économiques, d'une composition conçue pour une coupole. En effet, un oculus ou clipeus central, avec ou sans image, se trouve au sommet de nombreuses coupoles depuis l'Antiquité; il suffit de penser au Panthéon de Rome. Mais encore plus importante est la présence très fréquente d'une image centrale dans les coupoles des baptistères paléochrétiens, cette dernière étant souvent séparée par un encadrement circulaire du reste de la décoration de la coupole ${ }^{42}$. Ainsi, nous trouvons la scène du baptême du Christ disposée dans le médaillon central du Baptistère des Orthodoxes et au Baptistère des Ariens à Ravenne, ou l'image du staurogramme doré entouré d'une couronne de laurier au baptistère napolitain San Giovanni in Fonte. Ce baptistère partage un élément de plus avec la mosaïque d'Albenga: le ciel bleu foncé parsemé d'étoiles qui englobe le monogramme lumineux du Christ. Le motif de la croix ou du monogramme du Christ apparaissant au milieu du ciel nocturne se retrouve d'ailleurs dans d'autres coupoles de l'Antiquité tardive, par exemple au "Mausolée» de Galla Placidia à Ravenne ou à Santa Maria de Casaranello dans les Pouilles. Laissant de côté la valeur de la coupole comme allusion sémantique à la sphère

4I. M. Marcenaro, Il battistero "monumentale» di Albenga, p. 64 sq.

42. Voir l'article de M. Braconi dans ce même volume. 
Fig. 6 - Restitution hypothétique de la mosaïque de la coupole du baptistère San Giovanni alle Fonti.

céleste, il est évident que l'image du ciel étoilé trouve sa place par excellence dans cet espace sphérique.

À la lumière de ces considérations, nous avons tenté d'imaginer le motif central de la voûte à Albenga, déplacé et déployé dans une coupole (fig. 6). Nous avons choisi pour cadre architectural le baptistère de Fréjus, en Provence, qui appartient au groupe de baptistères de la zone du nord de l'Italie et de la Provence, datant tous du $\mathrm{V}^{\mathrm{e}}$ et $\mathrm{VI}^{\mathrm{e}}$ siècle et qui partagent un plan architectural très similaire avec le baptistère milanais ${ }^{43}$. Même si le baptistère de Fréjus présente des dimensions nettement inférieures au baptistère de Milan, il est l'un des rares édifices du groupe susmentionné qui conserve presque intacte sa structure murale originale, y compris la coupole. Comme mentionné, de nombreuses tesselles en verre ont été découvertes dans les vestiges du baptistère milanais. Une large portion d'entre elles étaient dorées et c'est pourquoi il serait opportun d'imaginer qu'au-delà du chrisme central, très certainement doré, l'or était une couleur dominante dans la coupole, remplaçant peut-être le ciel bleu foncé étoilé.

43. Cf. M. Sannazaro, "L'edificio battesimale nella metropoli milanese e nelle diocesi suffraganee lombarde»; J. Guyon, Les premiers baptistères des Gaules (IVe-VIII e siècles). 
Mis à part les dimensions de la décoration originelle et l'apparence de la surface qui entourait le clipeus central dans la coupole de San Giovanni alle Fonti, nous rencontrons un problème qui reste difficile à résoudre en l'état actuel de nos connaissances. Il s'agit de la datation de l'élément central de la mosaïque dont nous avons tenté de restituer l'iconographie. Comme indiqué, le baptistère milanais a connu deux phases d'édification importantes, dont la seconde, datée du tournant du $\mathrm{VI}^{\mathrm{e}}$ siècle, aurait inclus une nouvelle décoration en mosaïque. Le commanditaire de ces interventions, l'évêque Laurent $\mathrm{I}^{\mathrm{er}}$, est responsable d'une véritable renovatio de l'église milanaise et de ses édifices sacrés; mis à part la reconstruction du baptistère, on peut selon toute vraisemblance lui assigner la reconstruction de la chapelle Saint-Victor-au-ciel-d'or près de la basilique ambrosienne et les interventions d'embellissement de la zone de l'autel de la même basilique qui ont suivi la reconnaissance de la tombe d'Ambroise, de Gervais et de Protais qui se trouvait au-dessous de l'autel ${ }^{44}$. L'évêque Laurent a donc également été un promoteur du culte des saints locaux milanais et, chose plus importante encore, en s'intéressant à ses prédécesseurs à la chaire épiscopale milanaise, et à Ambroise en particulier, il a posé des bases pour le développement du culte de ce saint évêque ${ }^{45}$. C'est pourquoi il est à notre avis possible que la mosaïque ait pu être une spolia concettuale ${ }^{46}$, c'est-à-dire une reprise de la décoration précédente, peut-être ambrosienne, ou au moins directement inspirée par les explications théologiques du baptême qu’a laissées Ambroise à la postérité. La dimension antiarienne que Mario Marcenaro voit dans la composition d'Albenga prendrait dans ce cas également tout son sens: qui d'autre, sinon le grand ennemi des Ariens, Ambroise, aurait pu concevoir une telle image?

\section{Alžběta FiLIPová Tbilisi State University, Art History and Theory Department}

44. Cf. I. Foletti, «Le tombeau d'Ambroise».

45. Cf. I. Foletti, Oggetti, reliquie e migranti.

46. Concept utilisé par Serena Romano pour la décoration de l'abside de l'ancienne basilique de Saint-Pierre à Rome. Cf. M. Andaloro, S. Romano, «L'immagine nell'abside», p. 115. 


\section{BIBLIOGRAPHIE}

\section{Sources}

Ambroise de Milan, Épîtres - Opera omnia di Sant'Ambrogio, Lettere, vol. 19-21, a cura di Gabriele Banterle, Milano, Roma, Città nuova editrice, 1988.

-, De sacramentis, De mysteriis, Explanatio symboli - Des sacrements. Des mystères, Explication du symbole, éd. par Dom Bernard Botte, Paris, Cerf, 1994 (2 $2^{\mathrm{e}}$ impression de la nouvelle édition).

Ennode de Pavie, Carmina, Magni Felici Ennodi opera, éd. par Guillaume Hartel, CSEL 6, Vindobonae, 1882.

\section{Études}

Andaloro, Maria, Romano, Serena, "L'immagine nell'abside», in Arte e iconografia a Roma: da Costantino a Cola di Rienzo, a cura di Maria Andaloro, Serena Romano, Milano, Jaca Book, 2000, p. 93-132.

Ayres, Lewis, Nicaea and its legacy: an approach to fourth-century trinitarian theology, Oxford, Oxford University Press, 2006.

Bertelli, Carlo, "Mosaici a Milano", in Milano e i milanesi prima del Mille (VIII-X secolo). Atti del X Congresso internazionale di studi sull'Alto Medioevo (26-30 settembre 1983), Spoleto, Centro italiano di studi sull'alto medioevo, 1986, p. 333-351.

Brandt, Olof et al., "Photo modelling as an instrument for stratigraphic analysis of standing buildings: the baptistery of Albenga", Rivista di archeologia cristiana, 90 (2014), p. 259-293.

Cattaneo, Enrico, Terra di Sant'Ambrogio. La chiesa di Milano nel primo millennio, Milano, Vita e pensiero, 1989.

Clark, Gillian, "Victricius of Rouen: Praising the Saints", Journal of Early Christian Studies, 7 (1999), p. 365-399. 
Dennis, Nathan S., Performing paradise in the early Christian baptistery: art, liturgy, and the transformation of vision, thèse de doctorat dirigée par Herbert L. Kessler, soutenue à Johns Hopkins University en mai 2016.

-, "Bodies in motion: Visualizing Trinitarian space in the Albenga baptistery", in Perceptions of the body and sacred space in Late Antiquity and Byzantium, ed. by Jelena Bogdanović, London, Routledge, 2018, p. 124-148.

Fieni, Laura, "Il battistero di San Giovanni alle Fonti di Milano: un caso di studio archeologico-archeometrico", Archeologia dell'architettura, 3 (1998), p. 91-108.

FilipovÁ, Alžběta, "The circulation of blood, clay and ideas: the distribution of Milanese relics in the fourth and fifth centuries", Convivium, 1/1 (2014), p. 64-75.

-, Milan sans frontières. Le culte et la circulation des reliques milanaises et leur impact sur la diffusion de l'art et l'architecture de Milan pendant l'Antiquité tardive, Rome, Viella, sous presse.

Folettri, Ivan, «Le tombeau d'Ambroise: cinq siècles de construction identitaire", in L'évêque, l'image et la mort. Identité et mémoire au Moyen Âge, éd. par Nicolas Bock, Ivan Foletti, Michele Tomasi, Rome, Viella, 2014, p. 73-101.

-, Oggetti, reliquie e migranti. La basilica ambrosiana e il culto dei suoi santi (386-972), Roma, Viella, 2018.

Franceschi, Enrico, Nole, Dion, Vassallo, Stefano, «Il mosaico del battistero di Albenga. Indagini in fluorescenza X (XRF) e altre tecniche non invasive", in Ravenna musiva. Conservazione e restauro del mosaico antico e contemporaneo, a cura di Cesare Fiore, Mariangela Vandini, Bologna, Ante quem, 2010, p. 483-496.

Gandolfi, Daniela, Frondoni, Alessandra, "Recenti indagini archeologiche nel battistero "monumentale" di Albenga. Note di scavo", in Albenga città episcopale. Tempi e dinamiche della cristianizzazione tra Liguria di Ponente e Provenza, a cura di Mario Marcenaro, Genova, Istituto internazionale di studi liguri, 2007, p. 555-598.

Gavrilyuk, Paul L., Coakley, Sarah (eds), The spiritual senses: perceiving God in western Christianity, Cambridge, Cambridge University Press, 2011. 
Guyon, Jean, Les premiers baptistères des Gaules (IVe-VIII siècles), Rome, Unione internazionale degli istituti di archeologia, storia e storia dell'arte in Roma, 2000.

IvanovicI, Vladimir, Manipulating theophany. Light and ritual in north Adriatic architecture (ca. 400-ca. 800), Berlin, Walter de Gruyter, 2016.

Johnson, Mark J., The Roman imperial mausoleum in Late Antiquity, Cambridge, Cambridge University Press, 2009.

Kinney, Dale, "Le chiese paleocristiane di Mediolanum", in Milano, una capitale da Ambrogio ai carolingi, a cura di Carlo Bertelli, Milano, Electa, 1987, p. 48-79.

LizzI, Rita, "Ambrose's contemporaries and the christianization of northern Italy", Journal of Roman Studies, 80 (1990), p. 156-173.

Lusuardi Siena, Silvia, "Il complesso episcopale», in Milano capitale dell'impero romano (286-402 d.C.). Catalogo della mostra (Milano 24 gennaio-22 aprile 1990), Cinisello Balsamo, Silvana Editoriale, 1990, p. 106-110.

—, «II complesso episcopale di Milano: riconsiderazione della testimonianza ambrosiana nella Epistola ad sororem ", Antiquité Tardive, 4 (1996), p. 124-132.

Lusuardi Siena, Silvia, Dellù, Elena, Delpiano, Maria Laura, Monti, Elena, "Lettura archeologica e prassi liturgica nei battisteri ambrosiani tra IV e VI secolo", Studia Ambrosiana, 6 (2012), p. 89-120.

Lusuardi Siena, Silvia, Sacchi, Furio, "Gli edifici battesimali di Milano e di Albenga", in Albenga città episcopale. Tempi e dinamiche della cristianizzazione tra Liguria di Ponente e Provenza, a cura di Mario Marcenaro, Genova, Istituto internazionale di studi liguri, 2007, p. 677-704.

Lusuardi Siena, Silvia, Sannazaro, Marco, «I battisteri del complesso episcopale milanese alla luce delle recenti indagini archeologiche», in L'edificio battesimale in Italia. Aspetti e problemi, Bordighera, Istituto internazionale di studi liguri, 2001, p. 647-674.

Marcenaro, Mario, "La cupola del Battistero di Albenga", Bizantinistica. Rivista di studi bizantini e slavi, 13 (2011), p. 61-92.

—, Il battistero "monumentale» di Albenga: sedici secoli di storia. Aggiornamento con appunti sulle recenti indagini archeologiche, Albenga, Edizioni del Delfino Moro, 2014. 
McLynn, Neil B., Ambrose of Milan: church and court in a Christian capital, Berkeley/London, University of California Press, 1994.

Neri, Elisabetta, Verità, Marco, Conventi, Alberto, "Glass mosaic tesserae from the 5th to 6th century baptistery of San Giovanni alle Fonti, Milan, Italy", in New light on old glass: recent research on Byzantine mosaics and glass, ed. by Chris Entwistle, Liz James, London, British Museum Press, 2013, p. 1-10.

Pighi, Giovanni B., "Commentariolus electorum», Aevum, 18 (1944), p. 14-51.

San Bernardino, Jesús, "Sub imperio discordiae: l'uomo che voleva essere Eliseo (giugno 386)", in Nec timeo mori, a cura di Luigi F. Pizzolato, Marco Rizzi, Milano, Vita e pensiero, 1998, p. 709-737.

Sannazaro, Marco "L'edificio battesimale nella metropoli milanese e nelle diocesi suffraganee lombarde", in Albenga città episcopale. Tempi e dinamiche della cristianizzazione tra Liguria di Ponente e Provenza, a cura di Mario Marcenaro, Genova, Istituto internazionale di studi liguri, 2007, p. 705-740.

Toesca, Pietro, La pittura e la miniatura nella Lombardia. Dai più antichi monumenti alla metà del Quattrocento, Milano, Ulrico Hoepli, 1912.

\section{Crédits iconographiques:}

Fig. 1 : d'après Mirabella Roberti, Mario, Paredi, Angelo, Il Battistero Ambrosiano di San Giovanni alle Fonti, Milano, Fabbrica del Duomo, 1974, p. 22.

Fig. 2: d'après Lusuardi Siena, Silvia, Sacchi, Furio, "Gli edifici battesimali di Milano e di Albenga", in Albenga città episcopale. Tempi e dinamiche della cristianizzazione tra Liguria di Ponente e Provenza, a cura di Mario Marcenaro, Genova, Istituto internazionale di studi liguri, 2007, p. 691.

Fig. 3-5: d'après Marcenaro, Mario, Il battistero monumentale di Albenga, sedici secoli di storia: aggiornamento con appunti sulle recenti indagini archeologiche, Albenga, Edizioni del Delfino Moro, 2014, p. 87, 132 et 129.

Fig. 6: (C) Alžběta Filipová, Kristýna Berta Marešová. 